\title{
Optimum Duration of Antibiotics in Acute Bacterial Meningitis: Where Do We Stand?
}

\author{
Nabaneeta Dash ${ }^{1} \cdot$ Jitendra Kumar Sahu ${ }^{2}$ (I) \\ Received: 4 January 2021 / Accepted: 19 January 2021 / Published online: 29 January 2021 \\ (C) Dr. K C Chaudhuri Foundation 2021
}

Acute bacterial meningitis (ABM) is one of the leading causes of mortality among young Indian children less than 5-y-old [1]. Consensus regarding the optimum duration of antibiotic therapy is lacking and is mostly based on identification of the causative agent and clinical progression of the disease. But the dilemma faced by most pediatricians in Indian sub-continent is lack of identification of the causative agent, either due to nonavailability of tests or due to prior use of antibiotics making CSF sterile. Most guidelines and standard textbook prescribe 10-14 d of antibiotics [2]. Shorter courses appear appealing, as this will not only reduce the cost of therapy but also decrease the incidence of health-care-associated infections.

The study by Vaswani and colleagues [3] adds to the growing evidence favoring shortening the duration of antibiotics in treatment of uncomplicated ABM in children. It is a wellplanned randomized trial comparing safety and efficacy of 7 vs $10 \mathrm{~d}$ of empiric antibiotic therapy in children ( 3 mo-14 y) with ABM. One hundred four children with confirmed or probable bacterial meningitis were randomized to receive either 7 or $10 \mathrm{~d}$ of intravenous ceftriaxone and vancomycin. These children were then followed up till $90 \mathrm{~d}$ after discharge. The study reported similar numbers of treatment failure (7vs 6), neurologic sequelae (6 vs 3) and relapse (7vs 6) in both groups.

The results of this study are encouraging. Though in the authors opinion, more clinical and laboratory data in the cases with treatment failure and relapse of meningitis, would be invaluable and add to the authors knowledge. Since treatment failure is the primary outcome in this study, it would be interesting to know the predisposing factors, clinical features and bacteriologic characteristics of these patients who did not

Jitendra Kumar Sahu jsh2003@gmail.com

1 Pediatric Infectious Disease Unit, Department of Pediatrics, Christian Medical College, Vellore, India

2 Pediatric Neurology Unit, Advanced Pediatrics Centre, Postgraduate Institute of Medical Education \& Research, Chandigarh, India improve despite $10 \mathrm{~d}$ of therapy. Smaller sample size and nonbacteriologically confirmed cases could be other impediments to the generalizability of the study outcomes.

Despite studies showing that shorter course of antimicrobials may be as effective as a longer course in the treatment of uncomplicated bacterial meningitis [4], most pediatricians as well as pediatric guidelines, still recommend 10-14 $\mathrm{d}$ of antibiotics in culture-negative cases. This is perhaps due to the apprehension regarding the persistence of Streptococcus pneumoniae bacteria, which is currently the most common cause of ABM [5]. Though other common organisms like $H$. influenzae and N. meningitidis require a shorter course of 7-10 d and 5-7 d, respectively. Hence, bacteriologic confirmation in the form of CSF culture or antigen testing or PCR or even positive blood culture with a pyogenic CSF picture, will not only help to tailor therapy duration according to the organism but also make it easier to distinguish viral from bacterial causes.

This study brings to fore the need to plan multicenter studies with a larger sample size to answer the question regarding safety and efficacy of shorter duration of empiric antibiotic therapy for the treatment of confirmed cases of ABM. Also, the study brings to notice the fact that meningitis remains a dreadful disease leaving behind sequalae in nearly $21 \%$ of the cases [3]. Therefore, preventive strategies like vaccination of young children, and catch-up vaccination of those unvaccinated, are crucial to prevent morbidity and mortality due to ABM.

\section{Compliance with Ethical Standards}

Conflict of Interest None.

\section{References}

1. Liu L, Chu Y, Oza S, et al. National, regional, and state-level allcause and cause-specific under-5 mortality in India in 2000-15: a systematic analysis with implications for the sustainable development goals. Lancet Glob Health. 2019;7(6):e721-34. 
2. Janowski AB, Hunstad DA. Acute bacterial meningitis beyond the neonatal period. In: Kliegman RM, St. Geme JW, Blum NJ, et al., editors. Nelson Textbook of Pediatrics, 21st ed. Philadelphia: Elsevier; 2019. p. 3223-4.

3. Vaswani ND, Gupta N, Yadav R, Nadda A. Seven versus ten days antibiotics course for acute pyogenic meningitis in children: a randomized controlled trial. Indian J Pediatr. 2020. https://doi.org/10. 1007/s12098-020-03454-1.

4. Molyneux E, Nizami SQ, Saha S, et al; CSF 5 Study Group. 5 versus 10 days of treatment with ceftriaxone for bacterial meningitis in children: a double-blind randomised equivalence study. Lancet. 2011;377(9780):1837-45.

5. Jayaraman Y, Veeraraghavan B, Chethrapilly Purushothaman GK, et al. Burden of bacterial meningitis in India: preliminary data from a hospital based sentinel surveillance network. PLoS One. 2018;13(5): e0197198.

Publisher's Note Springer Nature remains neutral with regard to jurisdictional claims in published maps and institutional affiliations. 